\title{
The last frontier for global non-communicable disease action: The emergency department-A cross-sectional study from East Africa
}

Christine Ngaruiya

Mbatha Wambua

Thomas Kedera Mutua

Daniel Owambo

Morgan Muchemi

See next page for additional authors

Follow this and additional works at: https://ecommons.aku.edu/eastafrica_fhs_mc_emerg_med

Part of the Emergency Medicine Commons 


\section{Authors}

Christine Ngaruiya, Mbatha Wambua, Thomas Kedera Mutua, Daniel Owambo, Morgan Muchemi, Kipkoech Rop, Kaitlin R. Maciejewski, Rebecca Leff, Mugane Mutua, and Benjamin Wachira 


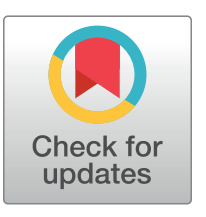

\section{G openaccess}

Citation: Ngaruiya C, Wambua M, Mutua TK, Owambo D, Muchemi M, Rop K, et al. (2021) The last frontier for global non-communicable disease action: The emergency department-A crosssectional study from East Africa. PLoS ONE 16(4): e0248709. https://doi.org/10.1371/journal. pone.0248709

Editor: Chaisiri Angkurawaranon, Chiang Mai University Faculty of Medicine, THAILAND

Received: September 20, 2020

Accepted: March 4, 2021

Published: April 2, 2021

Copyright: @ 2021 Ngaruiya et al. This is an open access article distributed under the terms of the Creative Commons Attribution License, which permits unrestricted use, distribution, and reproduction in any medium, provided the original author and source are credited.

Data Availability Statement: All relevant data are within the manuscript and its Supporting Information files.

Funding: The authors disclose receipt of the following financial support for the research, authorship, and publication of this article: This work was supported by the Hecht-Albert Global Health Pilot Innovation Award for Junior Faculty, Global Health Leadership Institute, Yale University awarded to CN. The funders had no role in study

\section{The last frontier for global non-communicable disease action: The emergency department- A cross-sectional study from East Africa}

\author{
Christine Ngaruiya $\oplus^{1 *}$, Mbatha Wambua ${ }^{2}$, Thomas Kedera Mutua ${ }^{3}$, Daniel Owambo ${ }^{4}$, \\ Morgan Muchemi ${ }^{5}$, Kipkoech Rop ${ }^{6}$, Kaitlin R. Maciejewski ${ }^{7}$, Rebecca Leff ${ }^{1,8}$, \\ Mugane Mutua ${ }^{9}$, Benjamin Wachira $^{10}$ \\ 1 Department of Emergency Medicine, Yale University, New Haven, CT, United States of America, \\ 2 Department of Emergency Medicine, Muhimbili University of Health and Allied Sciences, Dar-es-Salaam, \\ Tanzania, 3 The Nairobi West Hospital, Nairobi, Kenya, 4 The Aga Khan University Hospital, Nairobi, Kenya, \\ 5 Kenyatta University Teaching, Referral \& Research Hospital, Nairobi, Kenya, 6 University of Nairobi, \\ Nairobi, Kenya, 7 Yale Center for Analytical Sciences, Yale School of Public Health, New Haven, CT, United \\ States of America, 8 School of Medicine, Faculty of Health Sciences, Ben-Gurion University of the Negev, \\ Beer -Sheva, Israel, 9 Elburgon, Kenya, 10 Accident and Emergency Department, The Aga Khan University \\ Hospital, Nairobi, Kenya \\ * Christine.ngaruiya@yale.edu
}

\section{Abstract}

\section{Introduction}

Deaths due to non-communicable diseases (NCDs) have surpassed those due to communicable diseases globally and are projected to do so in Africa by 2030. Despite demonstrated effectiveness in high-income country $(\mathrm{HIC})$ settings, the ED is a primary source of NCD care that has been under-prioritized in Africa. In this study, we assess the burden of leading NCDs and NCD risk factors in Kenyan Casualty Department patients to inform interventions targeting patients with NCDs in emergency care settings.

\section{Materials and methods}

Using the WHO STEPwise approach to surveillance (STEPS) tool and the Personal Health Questionnaire (PHQ-9), we conducted a survey of 923 adults aged 18 and over at Kenyatta National Hospital Emergency Department (KNH ED) between May-October 2018. Age, income, household size(t-test), sex, education, marital status, work status, and poverty status (chi-squared test or fisher's exact test) were assessed using descriptive statistics and analyzed using covariate-adjusted logistic analysis.

\section{Results}

Over a third of respondents had hypertension ( $35.8 \%, n=225 / 628), 18.3 \%$ had raised blood sugar or diabetes $(18.3 \%, n=61 / 333)$, and $11.7 \%$ reported having cardiovascular disease $(11.7 \%, n=90 / 769)$. Having lower levels of education was associated with tobacco use (OR $6.0,95 \% \mathrm{Cl} 2.808-12.618, \mathrm{p}<0.0001)$, while those with higher levels of education reported increased alcohol use (OR $0.620(95 \% \mathrm{Cl} 0.386-0.994, p=0.0472)$. While a predominant 
design, data collection and analysis, decision to publish, or preparation of the manuscript. Funder website: https://medicine.yale.edu/news-article/ 14993/

Competing interests: The authors have declared that no competing interests exist. proportion of respondents had had some form of screening for either hypertension $(80.3 \%$, $n=630 / 772)$, blood sugar $(42.6 \%, n=334 / 767)$ or cholesterol $(13.9 \%, n=109 / 766)$, the proportion of those on treatment was low, with the highest proportion being half of those diagnosed with hypertension reporting taking medication $(51.6 \%, n=116 / 225)$.

\section{Conclusions}

This study establishes the ED as a high-risk population with potential for high impact in East Africa, should targeted interventions be implemented. Comprehension of the unique epidemiology and characteristics of patients presenting to the ED is key to guide care in African populations.

\section{Introduction}

Non-Communicable Diseases (NCDs) annually constitute more than $70 \%$ of deaths worldwide [1]. Furthermore, current disease trends suggest worsening of the situation over the next decade, with the WHO projecting 55 million deaths from NCDs annually by 2030 [2]. NCDs have surpassed communicable diseases as the lead cause of death in all continents except Africa, where NCD-related deaths are nevertheless projected to surpass deaths from communicable diseases, maternal and perinatal conditions, and nutritional deficiencies by 2030 [1]. Eighty-percent of deaths from NCDs occur in low- and middle- income countries (LMICs) with the majority of these occurring prematurely [2].

The 2013-2020 WHO global action plan for NCDs highlights such targets as: reduction in premature mortality secondary to cardiovascular disease, cancer, diabetes, and chronic respiratory disease; reduction in harmful use of alcohol; reduction in prevalence of tobacco use; reduction in prevalence of raised blood pressure, and increased prevalence of eligible people on appropriate therapy for cardiovascular disease prevention [2]. Interventions in highincome countries (HICs) have demonstrated the effectiveness of the Emergency Department (ED) in addressing all of these targets, including tobacco cessation, alcohol cessation, and use of navigators to improve compliance among diabetics, among others [3-5]. The ED is an optimal setting for these and other novel interventions targeting NCDs.

The ED is also the primary site for presentation of patients with acute NCD-related complications (such as acute coronary syndrome, strokes, diabetic ketoacidosis or asthma exacerbations), mental illness and injury-related complaints [6]. All of these conditions require timely and effective management in order to mitigate long-term effects of disease. The importance of studying NCDs in the ED and developing high yield interventions to improve management of those presenting with NCD-related acute illness to prevent downstream effects, is paramount.

In this study, we assess the burden of leading NCDs and NCD risk factors in Kenyan Casualty Department patients, in order to inform development of hospital and clinical policies, educational interventions for practitioners on management of NCDs in the emergency setting, and novel interventions targeting patients with NCDs in the Casualty setting. This is the largest epidemiological study on NCDs in an African Emergency Department that we are aware of.

\section{Materials and methods}

\section{Study design}

This was a prospective, cross-sectional study using surveys administered by data collectors to patients accessing care in the ED of the largest tertiary referral hospital in Kenya and East 
Africa, Kenyatta National Hospital. The WHO Stepwise Approach to Surveillance (STEPS) tool was used to assess burden of NCDs (particularly hypertension, diabetes and cardiovascular disease in this study), as well as prevalence of NCD risk factors (tobacco and alcohol in this study), and the Personal Health Questionnaire (PHQ-9) was used to assess prevalence of depression $[7,8]$. These diseases and risk factors were prioritized given their relative contribution to the global burden of NCD related morbidity and mortality [1], as well as because of established effectiveness to affect these particular conditions in the ED as demonstrated in HIC settings [3-5]. The tools are both internationally validated and publicly available. A Swahili version of the STEPS tool was obtained from the Kenya National STEPS study team [11]. A Swahili version of the PHQ-9 that has been validated in a Kenyan population by Omoro et al [8] was used for the latter. Results from the PHQ-9 tool are presented elsewhere.

Two data collectors were hired to assist with administration of surveys. Local data collectors were used who were familiar with the patient population and spoke the native languages. The survey was verbally administered to account for potential barriers with illiteracy, with responses indicated on electronic tablets. Surveys were loaded on to the Kobo software platform for ease of use. The surveys were offered in English and in the national language, Kiswahili. All patients provided written informed consent. This study received approval from the Institutional Review Board at Yale University (IRB Protocol ID 2000022697) and from the Kenyatta National Hospital/ University of Nairobi Ethics Review Committee (study registration No. A\&E/034/201).

\section{Sample size and population}

Kenyatta National Hospital (KNH), located in Nairobi, is the lead referral hospital with a catchment of 3 million people across East Africa. It is the largest ED in East Africa seeing a wide variety of nationals including Kenyans and other East Africans, "medical tourists," and refugees from across Africa. This was the study site. An estimate for the total number of patients seen at the KNH ED in a 3-month period (2014-2015) is between 14,956 and 23, 951, according to Myers et al [9]. No additional estimate is available to date from the facility or in the literature. Based on this, we had a target sample size of 2,400 or $10 \%$ of the upper estimate of total number of presentations over that time period (3 months), in line with standard pilot proportions [10]. We aimed to recruit these 2,400 participants similarly during a 3-month period (May 2018-July 2018). However, coinciding with the onset of data collection for our study, national referral protocols changed resulting in a substantial drop in the patient volume at the KNH ED. Therefore, in lieu of our original sampling approach, we instead extended data collection to the entirety of the funding period (6 months) and used a convenience sample of patients that were collected on randomized days, and across randomized time blocks for data collection (0800-1200, 1200-1600, and 1600-2000), to help ensure data collection across different days and time periods for presentation to the ED. Using this approach, we recruited patients presenting to the ED during the extent of the study period (May 2018-October 2018), and included all patients meeting our inclusion criteria. Patients were recruited in the ED for involvement in the study either after triage, during the waiting period prior to being evaluated by a clinician or during the period of time after when they had already been assessed and were awaiting disposition (Fig 1). This approach was used to help minimize interruptions to workflow in the Department. Patients who agreed to participate were then interviewed in a room adjacent to the ED clinical space. All patients aged 18 years old or older were considered, which mirrored the age group used in the 2015 Kenya STEPS study [11]. Patients who refused or were unable to provide informed consent were excluded. 


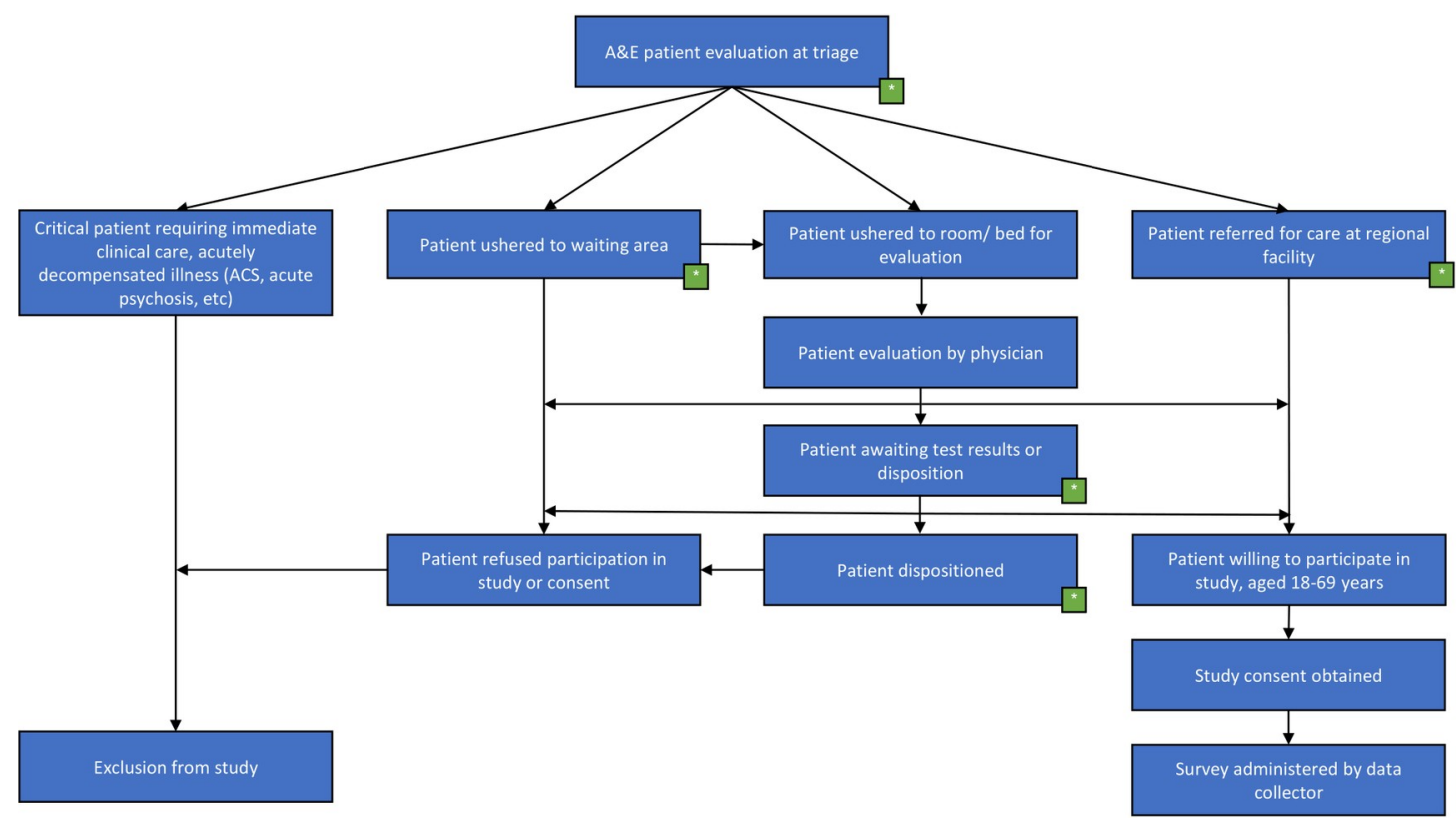

Fig 1. ED flow diagram/protocol. Key: A\&E-Accident \& Emergency Dept, ACS- Acute Coronary Syndrome, Green box-phase of care for approach by data collector.

https://doi.org/10.1371/journal.pone.0248709.g001

\section{Measures}

The analytical sample includes all those who consented to be surveyed. Not all participants answered all questions. Demographic information including age and sex (male, female) were collected. Marital status was categorized as married (currently married or cohabitating), formerly married (divorced, separated, widowed), never married. Participants were asked their highest education level, which was then categorized as secondary school or above (Secondary school completed, High school completed, College/University completed, Post graduate degree), primary school completed, less than primary school completed (less than primary school, no formal schooling). To collect employment information, participants were asked "which of the following best describes your main work status over the past 12 months?" Responses were then categorized as: Employed (Government employee, Non-government employee), Not employed (Homemaker, retired, non-paid, Unemployed (able to work), Unemployed (unable to work), Student, Self-employed.

To gain information about income, participants were asked "Taking the past year, can you tell me what the average earnings of the household have been?" They were also asked "How many people older than 18 years, including yourself, live in your household?" These variables were then used to create an indicator for household poverty, using the WHO standard [12]. The household was considered to be in poverty if household income/household size $<69397.5$ $\mathrm{KSH}$ (given a poverty cut-off of $\$ 1.90$ per person per day $(\$ 1.9 * 365.25$ days $* 100 \mathrm{KSh} / \$=$ 69397.5 KShs). Medication use was determined by asking "are you currently taking [medication]" for a given health disorder. The denominator was restricted only to those who were told they had the related condition by a doctor or other health worker.

\section{Statistical analysis}

First, descriptive statistics were generated to characterize the overall population. Mean, standard deviation, median, range are presented for numerical variables, percentages are presented 
for categorical variables, and number missing are presented for all. Bivariate analyses were next conducted for each outcome of interest. Variables were compared by age, income, household size (t-test), sex, education, marital status, work status, and poverty status (chi-squared test or fisher's exact test). Finally, multivariate analyses were conducted using covariateadjusted logistic analysis. $\mathrm{P}<0.05$ was considered statistically significant. A table is included with all statistically significant relationships and our outcomes of interest (Table 15). Analysis was conducted using SAS 9.4 (SAS Institute, Inc., Cary, NC).

\section{Results}

There were a total of 923 total respondents, of which $784(84.9 \%)$ provided consent. The mean age was $35 \mathrm{y}(+/-13.0 \mathrm{y})$, with a range from $18 \mathrm{y}$ to $88 \mathrm{y}$. The majority of respondents were male $(61.2 \%, \mathrm{n}=480 / 784)$ (see Table 1$)$. More than half of respondents had completed high school (secondary school) or higher $(59.4 \%, \mathrm{n}=466 / 784)$. The predominance of respondents reported being married $(54.6 \%, \mathrm{n}=428 / 784)$. Majority reported being self-employed $(41.2 \%$, $\mathrm{n}=323 / 784)$, and $19.9 \%$ reported being unemployed $(\mathrm{n}=156 / 784)$. The average reported annual household income was Kenya Shillings (Kshs) 237,888.6, or approximately 2,379 USD, with 30.6\% falling below the World Bank international poverty line of 1.90 USD per day $(\mathrm{n}=240 / 784)$.

More than a third of respondents reported being told they had elevated blood pressure or hypertension by a health worker $(35.8 \%, \mathrm{n}=225 / 628)$ (see Table 2$)$. The average age of those diagnosed was $41.7 \mathrm{y}(+/-14.5 \mathrm{y})$, with a female predominance $(56.25 \%, \mathrm{n}=126 / 225)$. The average reported income among those diagnosed was 2,406.85 USD, and most had completed high school (53.33\%, $\mathrm{n}=120 / 225)$ (see Tables 1 and 2). In the multivariate analysis, only age, sex, and being below the poverty line were predictors of likelihood of having been diagnosed with hypertension (Tables 3 and 15). For every 1-year increase in age, the odds of having been told by a doctor or other health worker that they had hypertension increased by $0.066(6.6 \%)(95 \%$ CI 1.041-1.092, $\mathrm{p}<0.0001)$. Women had more than double the odds of being told they had hypertension as compared to men (OR 2.335, 95\% CI 1.470-3.707, $\mathrm{p}=0.0003$ ). Those who were below the poverty line had $0.607(95 \%$ CI $0.372-0.992, \mathrm{p}=0.0462)$ times the odds of having been told by a doctor or other health worker that they had hypertension, compared to those who were not.

Nearly one in five patients $(18.3 \%, \mathrm{n}=61 / 333)$ reported being told they had elevated blood sugar or diabetes by a health worker (see Table 4 ). The average age among those reporting diagnosis was $49.5 y$ (+/- 13.9y). The mean reported income was 1,851.19 USD. The majority of respondents who reported having been diagnosed with diabetes were men $(60.7 \%, n=37 /$ $61)$, and tended towards lower levels of education with more than half $(63.9 \%, \mathrm{n}=39 / 61)$ reporting primary school or less. In the multivariate analysis, there was statistically significant evidence of likelihood of diagnosis being associated with advanced age (see Tables 5 and 15), and for every 1-year increase in age, the odds of having been told that they had raised blood sugar or diabetes increased by $0.072(7.2 \%)(95 \%$ CI 1.032-1.113, $\mathrm{p}=0.0003)$.

$38.5 \%$ (298/774) of respondents reported having ever used tobacco (smoked or smokeless), and $18.2 \%(n=119 / 654)$ of respondents reported being current smokers (see Table 1$)$. The average age of users was $38 \mathrm{y}$ with a range from $17 \mathrm{y}$ to $88 \mathrm{y}$, most had completed high school, and the vast majority of users were male (see Table 6). Male sex was a strong predictor of tobacco use, with an eight times (OR 8.86, 95\% CI 5.2-15.1) higher odds of use as compared to their female counterparts (see Tables 7 and 15). Additionally, lower levels of education were more predictive of tobacco use, with those having completed less than primary school being more likely to engage in tobacco use as compared to those that had completed secondary 
Table 1. Summary table on participants included in the study.

\begin{tabular}{|c|c|}
\hline & Total $(\mathrm{N}=784)$ \\
\hline \multicolumn{2}{|l|}{ Age } \\
\hline Missing (not Missing) & $17(767)$ \\
\hline Mean (SD) & $35.23(12.95)$ \\
\hline \multicolumn{2}{|l|}{ Household income } \\
\hline Missing (not Missing) & $268(516)$ \\
\hline Mean (SD) & $237888.61(268912.02)$ \\
\hline \multicolumn{2}{|l|}{ Household size } \\
\hline Missing (not Missing) & $9(775)$ \\
\hline Mean (SD) & $2.65(1.91)$ \\
\hline \multicolumn{2}{|l|}{ Sex } \\
\hline Missing & $2(0.26 \%)$ \\
\hline Female & $302(38.52 \%)$ \\
\hline Male & $480(61.22 \%)$ \\
\hline \multicolumn{2}{|l|}{ Education Level } \\
\hline Missing & $5(0.64 \%)$ \\
\hline Less than primary school or none & $102(13.01 \%)$ \\
\hline Primary school completed & $211(26.91 \%)$ \\
\hline Secondary school or above & $466(59.44 \%)$ \\
\hline \multicolumn{2}{|l|}{ Marital Status } \\
\hline Missing & $7(0.89 \%)$ \\
\hline Formerly Married & $68(8.67 \%)$ \\
\hline Married & $428(54.59 \%)$ \\
\hline Never married & $281(35.84 \%)$ \\
\hline \multicolumn{2}{|l|}{ Work Status } \\
\hline Missing & $8(1.02 \%)$ \\
\hline Employed & $202(25.77 \%)$ \\
\hline Self-employed & $323(41.20 \%)$ \\
\hline Student & $95(12.12 \%)$ \\
\hline Unemployed & $156(19.90 \%)$ \\
\hline \multicolumn{2}{|l|}{ Poverty } \\
\hline Missing & $269(34.31 \%)$ \\
\hline $\mathrm{N}$ & $275(35.08 \%)$ \\
\hline$Y$ & $240(30.61 \%)$ \\
\hline
\end{tabular}

Have you ever been told by a doctor or other health worker that you have raised blood pressure or hypertension?

\begin{tabular}{l|l} 
Missing & $156(19.90 \%)$ \\
\hline No & $403(51.40 \%)$ \\
\hline Yes & $225(28.70 \%)$ \\
\hline
\end{tabular}

Have you ever been told by a doctor or other health worker that you have raised blood sugar or diabetes?

\begin{tabular}{l|c}
\hline Missing & $451(57.53 \%)$ \\
\hline No & $272(34.69 \%)$ \\
\hline Yes & $61(7.78 \%)$
\end{tabular}

Have you ever had a heart attack or chest pain from heart disease (angina) or a stroke (cerebrovascular accident or incident)?

\begin{tabular}{l|c}
\hline Missing & $15(1.91 \%)$ \\
\hline No & $679(86.61 \%)$ \\
\hline Yes & $90(11.48 \%)$ \\
\hline
\end{tabular}

Have you ever been told by a doctor or other health worker that you have raised cholesterol? 
Table 1. (Continued)

\begin{tabular}{|c|c|}
\hline & Total $(\mathrm{N}=784)$ \\
\hline Missing & $675(86.10 \%)$ \\
\hline No & $79(10.08 \%)$ \\
\hline Yes & $30(3.83 \%)$ \\
\hline \multicolumn{2}{|c|}{ Ever used tobacco } \\
\hline Missing & $10(1.28 \%)$ \\
\hline No & $476(60.71 \%)$ \\
\hline Yes & $298(38.01 \%)$ \\
\hline \multicolumn{2}{|c|}{ Current tobacco user } \\
\hline Missing & $130(16.58 \%)$ \\
\hline No & $535(68.24 \%)$ \\
\hline Yes & $119(15.18 \%)$ \\
\hline \multicolumn{2}{|c|}{ Have you ever consumed any alcohol such as beer, wine, or other spirits? } \\
\hline Missing & $10(1.28 \%)$ \\
\hline No & $297(37.88 \%)$ \\
\hline Yes & $477(60.84 \%)$ \\
\hline \multicolumn{2}{|c|}{ Have you consumed any alcohol within the past 12 months? } \\
\hline Missing & $308(39.29 \%)$ \\
\hline No & $200(25.51 \%)$ \\
\hline Yes & $276(35.20 \%)$ \\
\hline \multicolumn{2}{|c|}{ During the past 30 days, did someone smoke in your home? } \\
\hline Missing & $10(1.28 \%)$ \\
\hline No & $458(58.42 \%)$ \\
\hline Yes & $316(40.31 \%)$ \\
\hline
\end{tabular}

During the past 30 days, did someone smoke in closed areas in your workplace (in the building, in a work area or a specific office)?

\begin{tabular}{l|r}
\hline Missing & $8(1.02 \%)$ \\
\hline Don't work in a closed area & $109(13.90 \%)$ \\
\hline No & $293(37.37 \%)$ \\
\hline Yes & $374(47.70 \%)$ \\
\hline
\end{tabular}

had your blood pressure measured by a doctor or other health worker?

\begin{tabular}{l|r}
\hline Missing & $12(1.53 \%)$ \\
\hline No & $142(18.11 \%)$ \\
\hline Yes & $630(80.36 \%)$ \\
\hline \multicolumn{2}{l|}{ Have you ever had your blood sugar measured by a doctor or other health worker? } \\
\hline Missing & $17(2.17 \%)$ \\
\hline No & $433(55.23 \%)$ \\
\hline Yes & $334(42.60 \%)$ \\
\hline
\end{tabular}

Have you ever had your cholesterol (fat levels in your blood) measured by a doctor or other health worker?

\begin{tabular}{l|r}
\hline Missing & $18(2.30 \%)$ \\
\hline No & $657(83.80 \%)$ \\
\hline Yes & $109(13.90 \%)$ \\
\hline
\end{tabular}

In the past two weeks, have you taken any drugs (medication) for raised blood pressure prescribed by a doctor or other health worker?

\begin{tabular}{l|r}
\hline Missing & $559(71.30 \%)$ \\
\hline No & $109(13.90 \%)$ \\
\hline Yes & $116(14.80 \%)$ \\
\hline Are you currently taking insulin for diabetes prescribed by a doctor or other health worker? \\
\hline
\end{tabular}

(Continued) 
Table 1. (Continued)

\begin{tabular}{|c|c|}
\hline & Total $(\mathrm{N}=784)$ \\
\hline Missing & $723(92.22 \%)$ \\
\hline No & $36(4.59 \%)$ \\
\hline Yes & $25(3.19 \%)$ \\
\hline \multicolumn{2}{|c|}{$\begin{array}{l}\text { In the past two weeks, have you taken any oral treatment (medication) for raised total cholesterol prescribed by } \\
\text { a doctor or other health worker? }\end{array}$} \\
\hline Missing & $754(96.17 \%)$ \\
\hline No & $19(2.42 \%)$ \\
\hline Yes & $11(1.40 \%)$ \\
\hline \multicolumn{2}{|c|}{ Are you currently taking aspirin regularly to prevent or treat heart disease? } \\
\hline Missing & $694(88.52 \%)$ \\
\hline No & $69(8.80 \%)$ \\
\hline Yes & $21(2.68 \%)$ \\
\hline \multicolumn{2}{|c|}{$\begin{array}{l}\text { Are you currently taking statins (Lovastatin/Simvastatin/Atorvastatin or any other statin) regularly to prevent } \\
\text { or treat heart disease? }\end{array}$} \\
\hline Missing & $698(89.03 \%)$ \\
\hline No & $71(9.06 \%)$ \\
\hline \multirow[t]{2}{*}{ Yes } & $15(1.91 \%)$ \\
\hline & Total $(\mathrm{N}=784)$ \\
\hline \multicolumn{2}{|c|}{ During the past 12 months, have you tried to stop smoking? } \\
\hline Missing & $680(86.73 \%)$ \\
\hline No & $45(5.74 \%)$ \\
\hline Yes & $59(7.53 \%)$ \\
\hline \multicolumn{2}{|c|}{$\begin{array}{l}\text { Have you stopped drinking due to health reasons, such as a negative impact on your health or on the advice of } \\
\text { your doctor or other health worker? }\end{array}$} \\
\hline Missing & $584(74.49 \%)$ \\
\hline No & $135(17.22 \%)$ \\
\hline Yes & $65(8.29 \%)$ \\
\hline
\end{tabular}

https://doi.org/10.1371/journal.pone.0248709.t001

school or above (OR 6.0, 95\% CI 2.808-12.618, $\mathrm{p}<0.0001$ ), adjusting for other covariates. $40.8 \%(\mathrm{n}=316 / 774)$ of respondents reported being exposed to smoke in home, and $47.7 \%$ reported exposure $(\mathrm{n}=374 / 776)$ at work.

Another 61.6\% (477/774) reported having used alcohol, majority being current users, having consumed alcohol within the past year. The average age of alcohol users was $36 \mathrm{y}$, and majority of users were also male (see Table 8). Females have 0.324 (95\% CI 0.215-0.488, p < .0001) times the odds of ever consuming any alcohol, compared to males, adjusting for other covariates (see Tables 9 and 15). Those who completed primary school have 0.620 (95\% CI $0.386-0.994, p=0.0472)$ times the odds, compared to those who attended secondary school or above.

Majority of respondents had either had their blood pressure (80.3\%, $n=630 / 772)$, blood sugar $(42.6 \%, \mathrm{n}=334 / 767)$ or cholesterol $(13.9 \%, \mathrm{n}=109 / 766)$ measured (see Tables $10-12)$. Of those that had been told they had elevated blood pressure, 51.6\% $(n=116 / 225)$ reported taking medications. Age was the only sociodemographic variable that had statistically significant evidence predicting likelihood of using antihypertensives (see Tables 13 and 15). For every one year increase in age, the odds of ever having taken medication for hypertension in the past two weeks increased by 0.089 (8.9\%) (95\% CI 1.044-1.136, p <0.0001), adjusting for other covariates. Furthermore, of those that had been told they had elevated blood sugar or diabetes, $41 \%(n=25 / 61)$ reported taking insulin. Again, age was the only predictor with 
Table 2. Sociodemographic characteristics of respondents told by a doctor or other health worker that they had raised blood pressure or hypertension.

\begin{tabular}{|c|c|c|c|c|}
\hline & No $(N=403)$ & Yes $(\mathrm{N}=225)$ & Total $(\mathrm{N}=628)$ & P Value \\
\hline \multicolumn{5}{|l|}{ Age } \\
\hline Mean (SD) & $33.57(11.82)$ & $41.69(14.48)$ & $36.51(13.41)$ & $<0.001^{* * *}$ \\
\hline \multicolumn{5}{|l|}{ Household income (Ksh) } \\
\hline Mean (SD) & $255320.72(298334.54)$ & $240685.90(276783.29)$ & $249711.30(290005.41)$ & 0.62 \\
\hline \multicolumn{5}{|l|}{ Household size } \\
\hline Mean (SD) & $2.60(2.03)$ & $2.96(2.04)$ & $2.73(2.04)$ & $0.034^{*}$ \\
\hline \multicolumn{5}{|l|}{ Sex } \\
\hline Female & $149(37.06 \%)$ & $126(56.25 \%)$ & $275(43.93 \%)$ & $<0.001^{* * *}$ \\
\hline Male & $253(62.94 \%)$ & $98(43.75 \%)$ & $351(56.07 \%)$ & \\
\hline \multicolumn{5}{|l|}{ Education Level } \\
\hline Less than primary school or none & $34(8.48 \%)$ & $45(20.00 \%)$ & $79(12.62 \%)$ & $<0.001^{* * *}$ \\
\hline Primary school completed & $107(26.68 \%)$ & $60(26.67 \%)$ & $167(26.68 \%)$ & \\
\hline Secondary school or above & $260(64.84 \%)$ & $120(53.33 \%)$ & $380(60.70 \%)$ & \\
\hline \multicolumn{5}{|l|}{ Marital Status } \\
\hline Formerly Married & $24(6.02 \%)$ & $31(13.78 \%)$ & $55(8.81 \%)$ & $<0.001^{* * *}$ \\
\hline Married & $219(54.89 \%)$ & $140(62.22 \%)$ & $359(57.53 \%)$ & \\
\hline Never married & $156(39.10 \%)$ & $54(24.00 \%)$ & $210(33.65 \%)$ & \\
\hline \multicolumn{5}{|l|}{ Work Status } \\
\hline Employed & $107(26.68 \%)$ & $53(23.77 \%)$ & $160(25.64 \%)$ & $0.038^{*}$ \\
\hline Self-employed & $157(39.15 \%)$ & $100(44.84 \%)$ & $257(41.19 \%)$ & \\
\hline Student & $56(13.97 \%)$ & $16(7.17 \%)$ & $72(11.54 \%)$ & \\
\hline Unemployed & $81(20.20 \%)$ & $54(24.22 \%)$ & $135(21.63 \%)$ & \\
\hline \multicolumn{5}{|l|}{ Poverty } \\
\hline No & $135(54.00 \%)$ & $79(50.64 \%)$ & $214(52.71 \%)$ & 0.51 \\
\hline Yes & $115(46.00 \%)$ & $77(49.36 \%)$ & $192(47.29 \%)$ & \\
\hline
\end{tabular}

https://doi.org/10.1371/journal.pone.0248709.t002

statistical evidence for association with taking insulin in diabetic patients, with the odds of use increasing with age (OR 1.3, 95\%CI 1.1-1.6, $\mathrm{p}=0.0115)$, adjusting for other covariates (see Tables 14 and 15). There were no statistically evident predictors of taking statins or aspirin. While more than one in ten respondents reported a history of cardiovascular disease-either angina, heart disease or stroke $(11.7 \%, \mathrm{n}=90 / 769)$, only one in four $(23.3 \%, \mathrm{n}=21 / 90)$ of

Table 3. Logistic regression analysis on respondents who reported being told that they had hypertension.

\begin{tabular}{|c|c|c|c|c|}
\hline \multicolumn{5}{|c|}{ Have you ever been told by a doctor or other health worker that you had hypertension? Yes vs No } \\
\hline \multicolumn{2}{|r|}{ Effect } & \multirow{2}{*}{\begin{tabular}{|c|} 
Odds Ratio Estimates \\
2.335 \\
\end{tabular}} & \multirow{2}{*}{\begin{tabular}{|c|} 
95\% Wald Confidence Limits \\
$(1.470,3.707)$ \\
\end{tabular}} & \multirow{2}{*}{\begin{tabular}{|c|} 
P-value \\
0.0003 \\
\end{tabular}} \\
\hline Sex. & Female vs Male & & & \\
\hline $\begin{array}{l}\text { Education } \\
\text { Level. }\end{array}$ & $\begin{array}{l}\text { Less than primary school or no formal schooling vs Secondary school or } \\
\text { above }\end{array}$ & 1.109 & $(0.492,2.500)$ & 0.80 \\
\hline $\begin{array}{l}\text { Education } \\
\text { Level. }\end{array}$ & Primary school completed vs Secondary school or above & 1.029 & $(0.599,1.767)$ & 0.92 \\
\hline Marital Status. & Formerly married vs Never married & 1.813 & $(0.702,4.678)$ & 0.22 \\
\hline Marital Status. & Married vs Never married & 1.175 & $(0.657,2.102)$ & 0.59 \\
\hline Work Status. & Employed vs Unemployed & 0.743 & $(0.350,1.576)$ & 0.44 \\
\hline Work Status. & Self-employed vs Unemployed & 0.630 & $(0.313,1.268)$ & 0.20 \\
\hline Work Status. & Student vs Unemployed & 0.931 & $(0.281,3.077)$ & 0.91 \\
\hline Age & Years & 1.066 & $(1.041,1.092)$ & $<.0001$ \\
\hline Poverty. & Yes vs No & 0.607 & $(0.372,0.992)$ & 0.046 \\
\hline
\end{tabular}

https://doi.org/10.1371/journal.pone.0248709.t003 
Table 4. Sociodemographic characteristics of respondents told by a doctor or other health worker that they had raised blood sugar or diabetes.

\begin{tabular}{|c|c|c|c|c|}
\hline & No $(N=272)$ & Yes $(N=61)$ & Total $(\mathrm{N}=333)$ & P Value \\
\hline \multicolumn{5}{|l|}{ Age } \\
\hline Mean (SD) & $38.38(13.42)$ & $49.48(13.89)$ & $40.43(14.16)$ & $<0.001^{* * *}$ \\
\hline \multicolumn{5}{|l|}{ Household income (Ksh) } \\
\hline Mean (SD) & $265718.23(285027.30)$ & $185119.05(211180.68)$ & $250538.12(274052.37)$ & $0.041^{*}$ \\
\hline \multicolumn{5}{|l|}{ Household size } \\
\hline Mean (SD) & $2.62(1.50)$ & $3.44(2.77)$ & $2.77(1.83)$ & $0.027^{*}$ \\
\hline \multicolumn{5}{|l|}{ Sex } \\
\hline Female & $125(45.96 \%)$ & $24(39.34 \%)$ & $149(44.74 \%)$ & 0.35 \\
\hline Male & $147(54.04 \%)$ & $37(60.66 \%)$ & $184(55.26 \%)$ & \\
\hline \multicolumn{5}{|l|}{ Education Level } \\
\hline Less than primary school or none & $31(11.48 \%)$ & $20(32.79 \%)$ & $51(15.41 \%)$ & $<0.001^{* * *}$ \\
\hline Primary school completed & $75(27.78 \%)$ & $19(31.15 \%)$ & $94(28.40 \%)$ & \\
\hline Secondary school or above & $164(60.74 \%)$ & $22(36.07 \%)$ & $186(56.19 \%)$ & \\
\hline \multicolumn{5}{|l|}{ Marital Status } \\
\hline Formerly Married & $30(11.15 \%)$ & $12(19.67 \%)$ & $42(12.73 \%)$ & 0.07 \\
\hline Married & $171(63.57 \%)$ & $40(65.57 \%)$ & $211(63.94 \%)$ & \\
\hline Never married & $68(25.28 \%)$ & $9(14.75 \%)$ & $77(23.33 \%)$ & \\
\hline \multicolumn{5}{|l|}{ Work Status } \\
\hline Employed & $73(27.04 \%)$ & $11(18.33 \%)$ & $84(25.45 \%)$ & $0.033^{*}$ \\
\hline Self-employed & $125(46.30 \%)$ & $33(55.00 \%)$ & $158(47.88 \%)$ & \\
\hline Student & $22(8.15 \%)$ & $0(0.00 \%)$ & $22(6.67 \%)$ & \\
\hline Unemployed & $50(18.52 \%)$ & $16(26.67 \%)$ & $66(20.00 \%)$ & \\
\hline \multicolumn{5}{|l|}{ Poverty } \\
\hline No & $102(56.67 \%)$ & $17(40.48 \%)$ & $119(53.60 \%)$ & 0.06 \\
\hline Yes & $78(43.33 \%)$ & $25(59.52 \%)$ & $103(46.40 \%)$ & \\
\hline
\end{tabular}

https://doi.org/10.1371/journal.pone.0248709.t004

these were taking aspirin and one in six $(17.44 \%, \mathrm{n}=15 / 86)$ were taking a statin. Only 3 CVD patients were taking both an aspirin and statin.

\section{Discussion}

In this study, we described the burden of NCDs and mental health at the Kenyatta National Hospital Emergency Department, the largest hospital in East Africa. More than a third of respondents had hypertension, one in five had raised blood sugar or diabetes, and more than one in ten reported having cardiovascular disease. More than one third reported tobacco use,

Table 5. Logistic regression analysis on respondents who reported being told that they had raised blood sugar or diabetes.

\begin{tabular}{|c|c|c|c|c|}
\hline \multicolumn{5}{|c|}{ Have you ever been told by a doctor or other health worker that you have raised blood sugar or diabetes? Yes vs No } \\
\hline \multicolumn{2}{|r|}{ Effect } & \multirow{2}{*}{$\begin{array}{c}\text { Odds Ratio Estimates } \\
0.593 \\
\end{array}$} & \multirow{2}{*}{\begin{tabular}{|c|} 
95\% Wald Confidence Limits \\
$(0.262,1.346)$ \\
\end{tabular}} & \multirow{2}{*}{\begin{tabular}{|c|} 
P-value \\
0.21
\end{tabular}} \\
\hline Sex. & Female vs Male & & & \\
\hline $\begin{array}{l}\text { Education } \\
\text { Level. }\end{array}$ & $\begin{array}{l}\text { Less than primary school or no formal schooling vs Secondary school or } \\
\text { above }\end{array}$ & 2.672 & $(0.922,7.744)$ & 0.082 \\
\hline $\begin{array}{l}\text { Education } \\
\text { Level. }\end{array}$ & Primary school completed vs Secondary school or above & 1.251 & $(0.481,3.254)$ & 0.56 \\
\hline Marital Status. & Formerly married vs Never married & 0.921 & $(0.226,3.756)$ & 0.94 \\
\hline Marital Status. & Married vs Never married & 0.783 & $(0.258,2.372)$ & 0.64 \\
\hline Age. & Years & 1.072 & $(1.032,1.113)$ & 0.0003 \\
\hline Poverty. & Yes vs No & 0.947 & $(0.404,2.221)$ & 0.90 \\
\hline
\end{tabular}

https://doi.org/10.1371/journal.pone.0248709.t005 
Table 6. Sociodemographic characteristics of respondents who reported having used tobacco (smoked or smokeless).

\begin{tabular}{|c|c|c|c|c|}
\hline & No $(N=476)$ & Yes $(\mathrm{N}=298)$ & Total $(\mathrm{N}=774)$ & P Value \\
\hline \multicolumn{5}{|l|}{ Age } \\
\hline Mean (SD) & $33.39(12.14)$ & $38.24(13.69)$ & $35.28(12.97)$ & $<0.001^{* * *}$ \\
\hline \multicolumn{5}{|l|}{ Household income (Ksh) } \\
\hline Mean (SD) & $239245.95(278069.92)$ & $231780.91(249729.78)$ & $236286.18(266968.18)$ & 0.76 \\
\hline \multicolumn{5}{|l|}{ Household size } \\
\hline Mean (SD) & $2.69(1.58)$ & $2.58(2.36)$ & $2.65(1.92)$ & 0.48 \\
\hline \multicolumn{5}{|l|}{ Sex } \\
\hline Female & $251(52.95 \%)$ & $46(15.44 \%)$ & $297(38.47 \%)$ & $<0.001^{* * *}$ \\
\hline Male & $223(47.05 \%)$ & $252(84.56 \%)$ & $475(61.53 \%)$ & \\
\hline \multicolumn{5}{|l|}{ Education Level } \\
\hline Less than primary school or none & $37(7.79 \%)$ & $65(21.89 \%)$ & $102(13.21 \%)$ & $<0.001^{* * *}$ \\
\hline Primary school completed & $123(25.89 \%)$ & $87(29.29 \%)$ & $210(27.20 \%)$ & \\
\hline Secondary school or above & $315(66.32 \%)$ & $145(48.82 \%)$ & $460(59.59 \%)$ & \\
\hline \multicolumn{5}{|l|}{ Marital Status } \\
\hline Formerly Married & $32(6.77 \%)$ & $36(12.12 \%)$ & $68(8.83 \%)$ & $0.033^{*}$ \\
\hline Married & $262(55.39 \%)$ & $161(54.21 \%)$ & $423(54.94 \%)$ & \\
\hline Never married & $179(37.84 \%)$ & $100(33.67 \%)$ & $279(36.23 \%)$ & \\
\hline \multicolumn{5}{|l|}{ Work Status } \\
\hline Employed & $122(25.74 \%)$ & $77(26.01 \%)$ & $199(25.84 \%)$ & $<0.001^{* * *}$ \\
\hline Self-employed & $197(41.56 \%)$ & $123(41.55 \%)$ & $320(41.56 \%)$ & \\
\hline Student & $76(16.03 \%)$ & $19(6.42 \%)$ & $95(12.34 \%)$ & \\
\hline Unemployed & $79(16.67 \%)$ & $77(26.01 \%)$ & $156(20.26 \%)$ & \\
\hline \multicolumn{5}{|l|}{ Poverty } \\
\hline No & $159(51.62 \%)$ & $113(55.67 \%)$ & $272(53.23 \%)$ & 0.37 \\
\hline Yes & 149 (48.38\%) & $90(44.33 \%)$ & 239 (46.77\%) & \\
\hline
\end{tabular}

https://doi.org/10.1371/journal.pone.0248709.t006

and two thirds reported alcohol use. Majority reported not taking medications despite diagnosis, with the highest proportion being half of those diagnosed with hypertension reporting taking medication. Determinants of disease burden were age, sex, and income. Having lower levels of education was associated with tobacco use, however those with higher levels of

Table 7. Logistic regression analysis on respondents who reported having used tobacco.

\begin{tabular}{|c|c|c|c|c|}
\hline \multicolumn{5}{|c|}{ Have you ever used tobacco? Yes vs No } \\
\hline \multicolumn{2}{|r|}{ Effect } & \multirow{2}{*}{$\begin{array}{c}\text { Odds Ratio Estimates } \\
0.117 \\
\end{array}$} & \multirow{2}{*}{\begin{tabular}{|c|} 
95\% Wald Confidence Limits \\
$(0.069,0.199)$
\end{tabular}} & \multirow{2}{*}{$\begin{array}{l}\text { P-value } \\
<.0001 \\
\end{array}$} \\
\hline Sex. & Female vs Male & & & \\
\hline $\begin{array}{l}\text { Education } \\
\text { Level. }\end{array}$ & $\begin{array}{l}\text { Less than primary school or no formal schooling vs Secondary school or } \\
\text { above }\end{array}$ & 5.952 & $(2.808,12.618)$ & $<.0001$ \\
\hline $\begin{array}{l}\text { Education } \\
\text { Level. }\end{array}$ & Primary school completed vs Secondary school or above & 1.472 & $(0.9,2.407)$ & 0.12 \\
\hline Marital Status. & Formerly married vs Never married & 1.083 & $(0.435,2.697)$ & 0.86 \\
\hline Marital Status. & Married vs Never married & 1.059 & $(0.625,1.795)$ & 0.83 \\
\hline Work Status. & Employed vs Unemployed & 0.573 & $(0.272,1.209)$ & 0.14 \\
\hline Work Status. & Self-employed vs Unemployed & 0.594 & $(0.295,1.197)$ & 0.15 \\
\hline Work Status. & Student vs Unemployed & 0.362 & $(0.127,1.028)$ & 0.056 \\
\hline Age & Years & 1.020 & $(0.999,1.042)$ & 0.059 \\
\hline Poverty. & Yes vs No & 0.664 & $(0.423,1.04)$ & 0.074 \\
\hline
\end{tabular}

https://doi.org/10.1371/journal.pone.0248709.t007 
Table 8. Sociodemographic characteristics of respondents who reported having consumed any alcohol within the past 12 months.

\begin{tabular}{|c|c|c|c|c|}
\hline & No $(N=200)$ & Yes $(\mathrm{N}=276)$ & Total $(\mathrm{N}=476)$ & P Value \\
\hline \multicolumn{5}{|l|}{ Age } \\
\hline Mean (SD) & $41.31(13.78)$ & $32.24(10.40)$ & $36.04(12.74)$ & $<0.001^{* * *}$ \\
\hline \multicolumn{5}{|l|}{ Household income (Ksh) } \\
\hline Mean (SD) & $245283.39(257736.17)$ & $247225.64(262612.57)$ & $246403.92(260177.74)$ & 0.95 \\
\hline \multicolumn{5}{|l|}{ Household size } \\
\hline Mean (SD) & $2.85(1.81)$ & $2.45(2.21)$ & $2.62(2.06)$ & $0.033^{*}$ \\
\hline \multicolumn{5}{|l|}{ Sex } \\
\hline Female & $69(34.50 \%)$ & $53(19.27 \%)$ & $122(25.68 \%)$ & $<0.001^{* * *}$ \\
\hline Male & $131(65.50 \%)$ & $222(80.73 \%)$ & $353(74.32 \%)$ & \\
\hline \multicolumn{5}{|l|}{ Education Level } \\
\hline Less than primary school or none & $33(16.58 \%)$ & $32(11.59 \%)$ & $65(13.68 \%)$ & 0.11 \\
\hline Primary school completed & $52(26.13 \%)$ & $61(22.10 \%)$ & $113(23.79 \%)$ & \\
\hline Secondary school or above & $114(57.29 \%)$ & $183(66.30 \%)$ & $297(62.53 \%)$ & \\
\hline \multicolumn{5}{|l|}{ Marital Status } \\
\hline Formerly Married & $19(9.60 \%)$ & $21(7.61 \%)$ & $40(8.44 \%)$ & $<0.001^{* * *}$ \\
\hline Married & $137(69.19 \%)$ & $130(47.10 \%)$ & $267(56.33 \%)$ & \\
\hline Never married & $42(21.21 \%)$ & $125(45.29 \%)$ & $167(35.23 \%)$ & \\
\hline \multicolumn{5}{|l|}{ Work Status } \\
\hline Employed & $57(28.79 \%)$ & $73(26.45 \%)$ & $130(27.43 \%)$ & $0.012^{*}$ \\
\hline Self-employed & $85(42.93 \%)$ & $117(42.39 \%)$ & $202(42.62 \%)$ & \\
\hline Student & $10(5.05 \%)$ & $38(13.77 \%)$ & $48(10.13 \%)$ & \\
\hline Unemployed & $46(23.23 \%)$ & $48(17.39 \%)$ & $94(19.83 \%)$ & \\
\hline \multicolumn{5}{|l|}{ Poverty } \\
\hline No & $67(46.85 \%)$ & $121(62.05 \%)$ & $188(55.62 \%)$ & $0.005^{* *}$ \\
\hline Yes & $76(53.15 \%)$ & $74(37.95 \%)$ & $150(44.38 \%)$ & \\
\hline
\end{tabular}

https://doi.org/10.1371/journal.pone.0248709.t008

education reported alcohol use. While a predominant proportion of respondents had had some form of screening for either hypertension, diabetes, or high cholesterol, the proportion of those on treatment was low. Our results showed a systematically higher NCD burden among the ED population as compared to the national population as demonstrated by the

Table 9. Logistic regression analysis on respondents who reported having ever consumed alcohol.

\begin{tabular}{|c|c|c|c|c|}
\hline \multicolumn{5}{|c|}{ Have you ever consumed any alcohol? Yes vs No } \\
\hline \multicolumn{2}{|r|}{ Effect } & \multirow{2}{*}{$\begin{array}{c}\text { Odds Ratio Estimates } \\
0.324 \\
\end{array}$} & \multirow{2}{*}{\begin{tabular}{|c|} 
95\% Wald Confidence Limits \\
$(0.215,0.488)$
\end{tabular}} & \multirow{2}{*}{$\begin{array}{l}\text { P-valu } \\
<.000 \\
\end{array}$} \\
\hline Sex. & Female vs Male & & & \\
\hline $\begin{array}{l}\text { Education } \\
\text { Level. }\end{array}$ & $\begin{array}{l}\text { Less than primary school or no formal schooling vs Secondary school or } \\
\text { above }\end{array}$ & 1.340 & $(0.658,2.728)$ & 0.42 \\
\hline $\begin{array}{l}\text { Education } \\
\text { Level. }\end{array}$ & Primary school completed vs Secondary school or above & 0.620 & $(0.386,0.994)$ & 0.047 \\
\hline Marital Status. & Formerly married vs Never married & 0.887 & $(0.395,1.994)$ & 0.77 \\
\hline Marital Status. & Married vs Never married & 1.535 & $(0.933,2.525)$ & 0.092 \\
\hline Work Status. & Employed vs Unemployed & 1.028 & $(0.527,2.007)$ & 0.94 \\
\hline Work Status. & Self-employed vs Unemployed & 1.120 & $(0.602,2.084)$ & 0.72 \\
\hline Work Status. & Student vs Unemployed & 0.885 & $(0.358,2.186)$ & 0.79 \\
\hline Age & Years & 1.010 & $(0.99,1.031)$ & 0.33 \\
\hline Poverty. & Yes vs No & 0.836 & $(0.551,1.269)$ & 0.40 \\
\hline
\end{tabular}

https://doi.org/10.1371/journal.pone.0248709.t009 
Table 10. Sociodemographic characteristics of respondents who reported having ever had their blood pressure measured by a doctor or other health worker.

\begin{tabular}{|c|c|c|c|c|}
\hline & No $(N=142)$ & Yes $(\mathrm{N}=630)$ & Total $(\mathrm{N}=772)$ & P Value \\
\hline \multicolumn{5}{|l|}{ Age } \\
\hline Mean (SD) & $29.70(8.68)$ & $36.53(13.43)$ & $35.25(12.95)$ & $<0.001^{* * *}$ \\
\hline \multicolumn{5}{|l|}{ Household income (Ksh) } \\
\hline Mean (SD) & $195892.40(164019.98)$ & $248629.58(289709.91)$ & $238102.79(270034.14)$ & $0.016^{*}$ \\
\hline \multicolumn{5}{|l|}{ Household size } \\
\hline Mean (SD) & $2.28(1.22)$ & $2.73(2.04)$ & $2.65(1.92)$ & $<0.001^{* * *}$ \\
\hline \multicolumn{5}{|l|}{ Sex } \\
\hline Female & $24(16.90 \%)$ & $275(43.79 \%)$ & $299(38.83 \%)$ & $<0.001^{* * *}$ \\
\hline Male & $118(83.10 \%)$ & $353(56.21 \%)$ & $471(61.17 \%)$ & \\
\hline \multicolumn{5}{|l|}{ Education Level } \\
\hline Less than primary school or none & $22(15.49 \%)$ & $79(12.58 \%)$ & $101(13.12 \%)$ & 0.55 \\
\hline Primary school completed & $40(28.17 \%)$ & $168(26.75 \%)$ & $208(27.01 \%)$ & \\
\hline Secondary school or above & $80(56.34 \%)$ & $381(60.67 \%)$ & $461(59.87 \%)$ & \\
\hline \multicolumn{5}{|l|}{ Marital Status } \\
\hline Formerly Married & $10(7.04 \%)$ & $56(8.95 \%)$ & $66(8.59 \%)$ & $0.009^{* *}$ \\
\hline Married & $65(45.77 \%)$ & $360(57.51 \%)$ & $425(55.34 \%)$ & \\
\hline Never married & $67(47.18 \%)$ & $210(33.55 \%)$ & $277(36.07 \%)$ & \\
\hline \multicolumn{5}{|l|}{ Work Status } \\
\hline Employed & $39(27.46 \%)$ & $160(25.56 \%)$ & $199(25.91 \%)$ & 0.14 \\
\hline Self-employed & $60(42.25 \%)$ & 259 (41.37\%) & $319(41.54 \%)$ & \\
\hline Student & $23(16.20 \%)$ & $72(11.50 \%)$ & $95(12.37 \%)$ & \\
\hline Unemployed & $20(14.08 \%)$ & $135(21.57 \%)$ & $155(20.18 \%)$ & \\
\hline \multicolumn{5}{|l|}{ Poverty } \\
\hline No & $57(55.88 \%)$ & $214(52.45 \%)$ & $271(53.14 \%)$ & 0.53 \\
\hline Yes & $45(44.12 \%)$ & $194(47.55 \%)$ & $239(46.86 \%)$ & \\
\hline
\end{tabular}

https://doi.org/10.1371/journal.pone.0248709.t010

Kenya national Stepwise approach to Surveillance (STEPs) study conducted 2 years prior [11] where the prevalence of hypertension was only in a quarter of the population, less than $3 \%$ had raised blood sugar or diabetes, only $13 \%$ reported tobacco use and 19\% reported alcohol use. The mental health results from our study will be presented elsewhere.

There was a higher prevalence of hypertension in our study on an ED population (35.8\%) as compared to the national STEPs study (24.5\%) utilizing the same validated survey tool [13], and as compared to the prevalence of national populations in the surrounding region: $24.3 \%$ in Uganda [14], 26\% in Tanzania [15], and 11.2\% in Rwanda [16]. The same is true for diabetes with only $2.1 \%$ of the general population reporting a diabetes diagnosis nationally in the Kenya STEPs study [11]. This high prevalence of disease in the ED population is well-established in the US, Canada and UK settings [17]. While the population presenting to the ED are likely to have higher prevalence of disease as this is a facility-based setting, and results may be biased by patients seeking care with known disease, this still establishes the ED as a high-risk population with potential for high-impact if targeted interventions are implemented, particularly in populations that may not otherwise access the healthcare system. A systematic review and meta-analysis by Armitage et al demonstrates the opportunity for detection of hypertension during screening in the ED [17]. This in turn provides the opportunity for patient education, linkage to care, or prescription of medication through the ED, and could affect immediate care such as screening for end-organ damage during the acute visit [4]. 
Table 11. Sociodemographic characteristics of respondents who reported having ever had their blood sugar measured by a doctor or other health worker.

\begin{tabular}{|c|c|c|c|c|}
\hline & No $(N=433)$ & Yes $(\mathrm{N}=334)$ & Total $(\mathrm{N}=767)$ & P Value \\
\hline \multicolumn{5}{|l|}{ Age } \\
\hline Mean (SD) & $31.19(10.11)$ & $40.43(14.14)$ & $35.18(12.85)$ & $<0.001^{* * *}$ \\
\hline \multicolumn{5}{|l|}{ Household income (Ksh) } \\
\hline Mean (SD) & $225626.40(257991.74)$ & $250538.12(274052.37)$ & $236562.06(265185.17)$ & 0.29 \\
\hline \multicolumn{5}{|l|}{ Household size } \\
\hline Mean (SD) & $2.55(1.99)$ & $2.77(1.82)$ & $2.64(1.92)$ & 0.11 \\
\hline \multicolumn{5}{|l|}{ Sex } \\
\hline Female & $148(34.34 \%)$ & $150(44.91 \%)$ & $298(38.95 \%)$ & $0.003^{* *}$ \\
\hline Male & $283(65.66 \%)$ & $184(55.09 \%)$ & $467(61.05 \%)$ & \\
\hline \multicolumn{5}{|l|}{ Education Level } \\
\hline Less than primary school or none & $50(11.55 \%)$ & $51(15.36 \%)$ & $101(13.20 \%)$ & 0.15 \\
\hline Primary school completed & $113(26.10 \%)$ & $95(28.61 \%)$ & $208(27.19 \%)$ & \\
\hline Secondary school or above & $270(62.36 \%)$ & $186(56.02 \%)$ & $456(59.61 \%)$ & \\
\hline \multicolumn{5}{|l|}{ Marital Status } \\
\hline Formerly Married & $24(5.56 \%)$ & $42(12.69 \%)$ & $66(8.65 \%)$ & $<0.001^{* * *}$ \\
\hline Married & $210(48.61 \%)$ & $212(64.05 \%)$ & $422(55.31 \%)$ & \\
\hline Never married & $198(45.83 \%)$ & $77(23.26 \%)$ & $275(36.04 \%)$ & \\
\hline \multicolumn{5}{|l|}{ Work Status } \\
\hline Employed & $114(26.33 \%)$ & $84(25.45 \%)$ & $198(25.95 \%)$ & $<0.001^{* * *}$ \\
\hline Self-employed & $159(36.72 \%)$ & $158(47.88 \%)$ & 317 (41.55\%) & \\
\hline Student & $72(16.63 \%)$ & $22(6.67 \%)$ & $94(12.32 \%)$ & \\
\hline Unemployed & $88(20.32 \%)$ & $66(20.00 \%)$ & $154(20.18 \%)$ & \\
\hline \multicolumn{5}{|l|}{ Poverty } \\
\hline No & $151(52.98 \%)$ & $119(53.60 \%)$ & $270(53.25 \%)$ & 0.89 \\
\hline Yes & $134(47.02 \%)$ & $103(46.40 \%)$ & 237 (46.75\%) & \\
\hline
\end{tabular}

https://doi.org/10.1371/journal.pone.0248709.t011

The average age of those diagnosed with hypertension was $42 y$. This is in contrast to USbased populations where hypertension is predominantly prevalent in those 60 years and older, as demonstrated by data from the National Health and Nutrition Examination Survey (NHANES) [18]. However, this observation of afflicted younger populations is not uncommon in African populations. Results from the Rwanda STEPS demonstrated a hypertension prevalence of 13\% among 25-34 year olds, and 19\% among 35-44 year olds [16]. The case is comparable for diabetes, with average age of diagnosis in our sample being $49 y$. Similarly, in the Rwanda case, the distribution of diabetes did not show significant difference for younger individuals. Our data support the need for greater attention to younger adults in this setting, as opposed to recommendations traditionally targeting only those 40 years and older [19]. By implementing primary and secondary prevention efforts for these younger groups the costly implications of NCD complications can be mitigated.

Women had a higher likelihood of hypertension diagnosis. The odds of women reporting a higher prevalence of disease is divergent from most African countries where there is a male predominance [20]. In the national STEPS study, men had much higher likelihood of not having had their blood pressure taken (71.1\%, 95\% CI 64.9-77.3) as compared to women (41.3\%, 95\% CI 36.5-46.0). Just as in our ED sample population, there was also a higher proportion of women that reported having been diagnosed as compared to men. Contrary to this, there was a predominance of men who reported having been diagnosed with diabetes (60.7\%) in our study, whereas in the national data, prevalence of disease was equal among both sexes. These 
Table 12. Have you ever had your cholesterol (fat levels in your blood) measured by a doctor or other health worker?.

\begin{tabular}{|c|c|c|c|c|}
\hline & No $(N=657)$ & Yes $(\mathrm{N}=109)$ & Total $(\mathrm{N}=766)$ & P Value \\
\hline \multicolumn{5}{|l|}{ Age } \\
\hline Mean (SD) & $33.95(12.06)$ & $42.74(15.33)$ & $35.16(12.91)$ & $<0.001^{* * *}$ \\
\hline \multicolumn{5}{|l|}{ Household income (Ksh) } \\
\hline Mean (SD) & $224326.85(248126.70)$ & $324800.00(364173.96)$ & $239160.48(270350.41)$ & $0.024^{*}$ \\
\hline \multicolumn{5}{|l|}{ Household size } \\
\hline Mean (SD) & $2.57(1.62)$ & $3.17(3.19)$ & $2.65(1.93)$ & 0.06 \\
\hline \multicolumn{5}{|l|}{ Sex } \\
\hline Female & $247(37.71 \%)$ & $51(46.79 \%)$ & $298(39.01 \%)$ & 0.07 \\
\hline Male & $408(62.29 \%)$ & $58(53.21 \%)$ & $466(60.99 \%)$ & \\
\hline \multicolumn{5}{|l|}{ Education Level } \\
\hline Less than primary school or none & $88(13.41 \%)$ & $13(12.04 \%)$ & $101(13.22 \%)$ & 0.53 \\
\hline Primary school completed & $180(27.44 \%)$ & $25(23.15 \%)$ & $205(26.83 \%)$ & \\
\hline Secondary school or above & $388(59.15 \%)$ & $70(64.81 \%)$ & $458(59.95 \%)$ & \\
\hline \multicolumn{5}{|l|}{ Marital Status } \\
\hline Formerly Married & $51(7.80 \%)$ & $14(12.96 \%)$ & $65(8.53 \%)$ & $0.001^{* *}$ \\
\hline Married & $350(53.52 \%)$ & $71(65.74 \%)$ & $421(55.25 \%)$ & \\
\hline Never married & $253(38.69 \%)$ & $23(21.30 \%)$ & $276(36.22 \%)$ & \\
\hline \multicolumn{5}{|l|}{ Work Status } \\
\hline Employed & $163(24.92 \%)$ & $33(30.56 \%)$ & $196(25.72 \%)$ & 0.37 \\
\hline Self-employed & $270(41.28 \%)$ & $46(42.59 \%)$ & $316(41.47 \%)$ & \\
\hline Student & $86(13.15 \%)$ & $9(8.33 \%)$ & $95(12.47 \%)$ & \\
\hline Unemployed & $135(20.64 \%)$ & $20(18.52 \%)$ & $155(20.34 \%)$ & \\
\hline \multicolumn{5}{|l|}{ Poverty } \\
\hline No & $226(52.31 \%)$ & $45(60.00 \%)$ & $271(53.45 \%)$ & 0.22 \\
\hline Yes & $206(47.69 \%)$ & $30(40.00 \%)$ & $236(46.55 \%)$ & \\
\hline
\end{tabular}

https://doi.org/10.1371/journal.pone.0248709.t012

findings highlight an interesting and important disparity in care-seeking behaviors of men, which may not occur unless symptoms exist. This is particularly detrimental for hypertension that tends to present asymptomatically [21]. To that end, increased attention is needed for screening and diagnosis of men during clinical encounters, including in the safety net of the ED while continuing to advance community-based prevention efforts.

Table 13. Logistic regression analysis on respondents who reported having taken medication for hypertension.

\begin{tabular}{|c|c|c|c|c|}
\hline \multicolumn{5}{|c|}{ In the past two weeks have you taken medication for hypertension? Yes vs No } \\
\hline \multicolumn{2}{|r|}{ Effect } & \multirow{2}{*}{\begin{tabular}{|c|} 
Odds Ratio Estimates \\
0.524 \\
\end{tabular}} & \multirow{2}{*}{\begin{tabular}{|c|} 
95\% Wald Confidence Limits \\
$(0.233,1.181)$
\end{tabular}} & \multirow{2}{*}{\begin{tabular}{|c|} 
P-value \\
0.12 \\
\end{tabular}} \\
\hline Sex. & Female vs Male & & & \\
\hline $\begin{array}{l}\text { Education } \\
\text { Level. }\end{array}$ & $\begin{array}{l}\text { Less than primary school or no formal schooling vs Secondary school or } \\
\text { above }\end{array}$ & 1.314 & $(0.381,4.538)$ & 0.67 \\
\hline $\begin{array}{l}\text { Education } \\
\text { Level. }\end{array}$ & Primary school completed vs Secondary school or above & 0.889 & $(0.353,2.241)$ & 0.80 \\
\hline Marital Status. & Formerly married vs Never married & 0.482 & $(0.108,2.145)$ & 0.34 \\
\hline Marital Status. & Married vs Never married & 0.621 & $(0.222,1.734)$ & 0.36 \\
\hline Work Status. & Employed vs Unemployed & 0.565 & $(0.158,2.027)$ & 0.38 \\
\hline Work Status. & Self-employed vs Unemployed & 0.854 & $(0.272,2.680)$ & 0.79 \\
\hline Work Status. & Student vs Unemployed & 0.332 & $(0.024,4.523)$ & 0.41 \\
\hline Age. & Years & 1.089 & $(1.044,1.136)$ & $<.0001$ \\
\hline Poverty. & Yes vs No & 1.254 & $(0.515,3.051)$ & 0.62 \\
\hline
\end{tabular}

https://doi.org/10.1371/journal.pone.0248709.t013 
Table 14. Logistic regression analysis on respondents who reported taking insulin.

\begin{tabular}{l|l|c|c|c}
\hline \multicolumn{1}{c}{ Are you currently taking insulin? Yes vs No } \\
\hline Sex. & Female vs Male & Odds Ratio Estimates & 95\% Confidence Limits & p-Value \\
\hline Education Level. & Less than primary school or no formal schooling vs Secondary school or above & 0.806 & $(0.095,6.862)$ & 0.84 \\
\hline Education Level. & Primary school completed vs Secondary school or above & 0.063 & $(0.001,2.779)$ & 0.15 \\
\hline Marital Status. & Formerly married vs Never married & 1.433 & $(0.07,29.185)$ & 0.81 \\
\hline Marital Status. & Married vs Never married & Years & 0.277 & $(0.007,11.35)$ \\
\hline Age. & Yes vs No & 0.061 & 0.50 \\
\hline Poverty. & & 1.32 & $(0.001,2.541)$ & 0.14 \\
\hline
\end{tabular}

https://doi.org/10.1371/journal.pone.0248709.t014

Those below the poverty line had $40 \%$ less likelihood of being diagnosed with hypertension. Additionally, among all patients that reported having a diagnosis of hypertension, diabetes, high cholesterol or CVD, majority had a secondary school education or higher. These markers of socioeconomic status raise concerns about diagnosis and management of disease among marginalized populations in the ED. The Lancet Commission on Reframing NCDs and Injuries (NCDI) for the Poorest Billion highlights poverty as a primary driver for NCDs [22]. In our study, the average reported annual household income was Kenya Shillings (Kshs) 237,888.6, or approximately 2,379 USD, with $30.6 \%$ falling below the World Bank international poverty line of 1.90 USD per day $(n=240 / 784)$. The Lancet Commission has facilitated development of "national NCDI poverty commissions", as well as leveraging the WHO PEN-Plus package-two interventions occurring at the policy level that could help facilitate sustainable change for individuals affected by NCDs in LMICs [23].

One in five Kenyans reported having used tobacco (21.3\%) in the national STEPS study [11], whereas $38.5 \%$ reported use in our study. Nearly half of individuals reported exposure to

Table 15. Sociodemographic factors that had statistically significant relationships with outcomes in regression analyses (outcomes: Presence of hypertension, raised blood sugar or diabetes, tobacco use, alcohol use, taking medications).

\begin{tabular}{|c|c|c|c|c|}
\hline & Effect & Odds Ratio Estimates & $\begin{array}{l}\text { 95\% Wald } \\
\text { Confidence Limits }\end{array}$ & P-value \\
\hline \multicolumn{5}{|c|}{ Have you ever been told by a doctor or other health worker that you had hypertension? Yes vs No } \\
\hline Sex. & Female vs Male & 2.335 & $(1.470,3.707)$ & 0.0003 \\
\hline Age. & Years & 1.066 & $(1.041,1.092)$ & $<.0001$ \\
\hline Poverty. & Yes vs No & 0.607 & $(0.372,0.992)$ & 0.046 \\
\hline \multicolumn{5}{|c|}{ Have you ever been told by a doctor or other health worker that you have raised blood sugar or diabetes? Yes vs No } \\
\hline $\begin{array}{l}\text { Education } \\
\text { Level. }\end{array}$ & $\begin{array}{l}\text { Less than primary school or no formal schooling vs Secondary school or } \\
\text { above }\end{array}$ & 2.672 & $(0.922,7.744)$ & 0.082 \\
\hline Age. & Years & 1.072 & $(1.032,1.113)$ & 0.0003 \\
\hline \multicolumn{5}{|c|}{ Have you ever used tobacco? Yes vs No } \\
\hline Sex. & Female vs Male & 0.117 & $(0.069,0.199)$ & $<.0001$ \\
\hline $\begin{array}{l}\text { Education } \\
\text { Level. }\end{array}$ & $\begin{array}{l}\text { Less than primary school or no formal schooling vs Secondary school or } \\
\text { above }\end{array}$ & 5.952 & $(2.808,12.618)$ & $<.0001$ \\
\hline \multicolumn{5}{|c|}{ Have you ever consumed any alcohol? Yes vs No } \\
\hline Sex. & Female vs Male & 0.324 & $(0.215,0.488)$ & $<.0001$ \\
\hline $\begin{array}{l}\text { Education } \\
\text { Level. }\end{array}$ & Primary school completed vs Secondary school or above & 0.620 & $(0.386,0.994)$ & 0.047 \\
\hline \multicolumn{5}{|c|}{ In the past two weeks have you taken medication for hypertension? Yes vs No } \\
\hline Age. & Years & 1.089 & $(1.044,1.136)$ & $<.0001$ \\
\hline \multicolumn{5}{|c|}{ Are you currently taking insulin? Yes vs No } \\
\hline Age. & Years & 1.32 & $(1.064,1.637)$ & 0.012 \\
\hline
\end{tabular}

https://doi.org/10.1371/journal.pone.0248709.t015 
smoke in the workplace or at home. In sum, exposure to tobacco is a significant problem in this population, and an alarming burden of disease is attributable to tobacco alone [24]. The WHO Tobacco Free Initiative highlights the role for brief tobacco interventions in healthcare settings, which have demonstrated effectiveness in US-based ED populations [5], but there are no studies to date on interventions in an African ED.

Overall, the prevalence of alcohol use was remarkably high. Those with higher levels of education were more likely to report engaging in alcohol use. This observation is divergent from tobacco use, which is more likely among those with lower levels of education. This phenomenon of education being associated with increased alcohol use has been demonstrated in other settings, including the US, however the downstream effects of alcohol use such as morbidity and mortality of disease remains disproportionately higher in those of lower socioeconomic status [25], and public health programming should be targeted accordingly. Brief interventions on alcohol intake in clinical settings are another intervention with demonstrated success in the ED setting [3].

Among those diagnosed with hypertension or diabetes, two of the leading NCDs, the vast majority were not taking medications for disease. In our study, the only determinant of using medications for NCDs and NCD risk factors was age. However, the lack of statistical evidence of differences in medication use compared across socioeconomic determinants likely indicates a universal lack of access regardless of sex, education and poverty level. Alternatively, it is possible that differences were not detected in some cases due to relatively small sample sizes for these questions. Our findings highlight the need to develop interventions to increase appropriate use of medication for individuals younger than 50y. Prescription of antihypertensives at ED discharge has been shown to improve blood pressure with no significant detrimental side effects in follow-up [26]. Mobilizing the ED to initiate, and help ensure compliance with, medications should be prioritized.

\section{Next steps}

Clinical protocols should be developed and routinely reviewed with providers, to help standardize therapy and empower education on self-care practices for patients. Education on hypertension and diabetes can also be facilitated by staff, and task-shifting can be employed such as through use of navigators-dedicated, trained nurses or lay health workers. Additionally, linkage to care programs such as those connecting to Community Health Workers could be implemented [27]. Mobile health (mHealth) technology presents a unique low-cost, highly effective opportunity to enhance health education, support self-monitoring, and improve follow-up [28], which could also be leveraged in ED populations. National policies that further address availability and affordability of medications are needed, in addition to ensuring enforcement of the WHO essential medicines list with first-line blood pressure and diabetes medications. In addition, efforts beyond the ED include media campaigns, and strengthening primary healthcare. WHO initiatives such as the SAFER technical package address alcohol access, and include strengthening restrictions on alcohol availability, enforcing restrictions on alcohol advertising and promotion, among other recommendations [29]. Finally, our findings highlight the importance of legislation that implements and enforces smoke-free zones in public places and workplaces, as well as regulates how these policies are enforced with clear sanctions for entities not abiding by guidelines [30].

\section{Limitations}

The sample studied in our population may not have findings generalizable to the national population given the study was conducted at a tertiary, referral hospital which may represent a population with higher prevalence of comorbidities. Also, given that this is a pilot study using a convenience sample, a nationally randomized control study would be beneficial to provide 
greater generalizability of results. Additionally, certain sub-analyses had too few respondents and so may have affected our ability to detect differences in this population, including the relative minority of women and our ability to detect further potential sex differences. With that said, in selecting a pilot study site, we felt that this site was likely to provide one of the most optimal sites to capture patients presenting from across the region, and our results provide novel evidence of concerning disease prevalence in a large population that seeks care here. Furthermore, our study provides insight into some of the most economically challenged populations in Kenya who receive care in the public health system, which is where the majority commonly seek care in Kenya and Africa at large. Finally, our findings provide incipient insights into a problem that has otherwise gone unaddressed, which will ideally generate opportunities for future research.

\section{Conclusions}

The ED acts as a catchment site for patients that may not otherwise frequent a healthcare setting. Comprehension of the unique epidemiology and characteristics of patients presenting to the ED is key in order to guide care. Opportunities exist for further research including longitudinal data collection through surveillance and registries to better understand epidemiology of disease, as well as implementation science to guide effective intervention planning. Patientdriven interventions, and collaboration with community-based stakeholders such as CHWs, would be ideal considerations to sustainably address NCDs leveraging the ED in the resourcelimited setting.

\section{Supporting information}

S1 File. STROBE checklist for cross-sectional studies. The STROBE checklist includes a list of items that should be included in reports of cross-sectional studies and is referred to in the Uniform Requirements for Manuscripts Submitted to Biomedical Journals by the International Committee of Medical Journal Editors.

(DOC)

\section{Acknowledgments}

The authors would like to acknowledge the contributions to data analysis by Mr. Manuel Martinez, Mr. Jesse Reynolds, and also would like to thank the Yale Center for Analytical Sciences (YCAS) support staff.

\section{Author Contributions}

Conceptualization: Christine Ngaruiya, Mbatha Wambua, Rebecca Leff, Benjamin Wachira.

Data curation: Christine Ngaruiya, Thomas Kedera Mutua, Daniel Owambo, Morgan Muchemi, Kipkoech Rop, Mugane Mutua.

Formal analysis: Christine Ngaruiya, Kaitlin R. Maciejewski.

Funding acquisition: Christine Ngaruiya.

Investigation: Thomas Kedera Mutua, Daniel Owambo, Morgan Muchemi, Kipkoech Rop, Mugane Mutua.

Methodology: Christine Ngaruiya, Kaitlin R. Maciejewski.

Project administration: Christine Ngaruiya. 
Resources: Thomas Kedera Mutua, Daniel Owambo, Morgan Muchemi, Kipkoech Rop, Mugane Mutua.

Supervision: Christine Ngaruiya.

Writing - original draft: Christine Ngaruiya, Mbatha Wambua, Rebecca Leff, Benjamin Wachira.

Writing - review \& editing: Christine Ngaruiya, Mbatha Wambua, Thomas Kedera Mutua, Daniel Owambo, Morgan Muchemi, Kipkoech Rop, Kaitlin R. Maciejewski, Rebecca Leff, Mugane Mutua, Benjamin Wachira.

\section{References}

1. World Health Organization. WHO Global status report on non-communicable diseases 2010: executive summary [Internet]. Geneva, Switzerland: World Health Organization; 2011 [Cited 2015 April 2]. Available from: http://apps.who.int/iris/bitstream/10665/44579/1/9789240686458_eng.pdf.

2. World Health Organization. Global action plan for the prevention and control of noncommunicable diseases 2013-2020 [Internet]. Geneva, Switzerland: World Health Organization; 2013 [Cited 2020 August 23]. Available from: https://www.who.int/nmh/events/ncd_action_plan/en/.

3. D'Onofrio G, Degutis LC. Preventive care in the emergency department: screening and brief intervention for alcohol problems in the emergency department: a systematic review. Acad Emerg Med. 2002; 9: 627-38. https://doi.org/10.1111/j.1553-2712.2002.tb02304.x PMID: 12045080

4. Irvin C, Wyer P, Gerson L. Preventive care in the emergency department, part II: clinical preventive services-an emergency medicine evidence-based review. Society for Academic Emergency Medicine Public Health and Education Task Force Preventive Services Work Group. Acad Emerg Med. 2000; 7: 1042-54. https://doi.org/10.1111/j.1553-2712.2000.tb02098.x PMID: 11044002

5. Lemhoefer C, Rabe GL, Wellmann J, et al. Emergency department-initiated tobacco control: update of a systematic review and meta-analysis of randomized controlled trials. Prev Chronic Dis. 2017; 14: E89. https://doi.org/10.5888/pcd14.160434 PMID: 28981403

6. Reynolds TA, Sawe H, Rubiano AM, et al. Strengthening Health Systems To Provide Emergency Care. 3rd ed. Washington (DC): The World Bank; 2017.

7. World Health Organization. STEPwise approach to surveillance (STEPS) [Internet]. Geneva, Switzerland: World Health Organization; 2016 [Cited 2016 February 1]. Available from: http://www.who.int/ chp/steps/en/.

8. Omoro SA, Fann JR, Weymuller EA, et al. Swahili translation and validation of the patient health questionnaire-9 depression scale in the Kenyan head and neck cancer patient population. Int J Psychiat Med. 2006; 36: 367-81. https://doi.org/10.2190/8W7Y-0TPM-JVGV-QW6M PMID: 17236703

9. Myers JG, Hunold KM, Ekernas K, et al. Patient characteristics of the accident and emergency department of Kenyatta National Hospital, Nairobi, Kenya: a cross-sectional, prospective analysis. BMJ Open. 2017; 7: e014974. https://doi.org/10.1136/bmjopen-2016-014974 PMID: 29025826

10. Baker TL. Doing Social Research. 2nd ed. New York: McGraw-Hill Inc; 1994.

11. Ministry of Health Republic of Kenya. Division of Noncommunicable Diseases. Kenya STEPwise survey for noncommunicable diseases risk factors 2015 report [Internet]. World Health Organization; 2015 [Cited 2020 May 1]. Available from: https:/www.health.go.ke/wp-content/uploads/2016/04/StepsReport-NCD-2015.pdf.

12. The World Bank. Understanding Poverty [Internet]. Washington (DC): The World Bank; 2020 [Cited 2017 September 1]. Available: https://www.worldbank.org/en/topic/poverty/overview2020.

13. Mohamed SF, Mutua MK, Wamai R, et al. Prevalence, awareness, treatment and control of hypertension and their determinants: results from a national survey in Kenya. BMC Public Health. 2018; 18 : 1219. https://doi.org/10.1186/s12889-018-6052-y PMID: 30400858

14. Ministry of Health Republic of Uganda. Uganda STEPS Survey 2014 Fact Sheet [Internet]. World Health Organization; 2014 [Cited 2020 May 1]. Available from: https://www.who.int/ncds/surveillance/ steps/Uganda_2014_STEPS_FactSheet.pdf.

15. Ministry of Health United Republic of Tanzania. Tanzania STEPS Survey 2012 Fact Sheet [Internet] World Health Organization; 2012 [Cited 2020 May 1]. Available from: https://www. who.int/ncds/ surveillance/steps/UR_Tanzania_FactSheet_2012.pdf. 
16. Republic of Rwanda Ministry of Health. Rwanda Non-communicable Diseases Risk Factors Report [Internet]. World Health Organization; 2015 [Cited 2020 May 1]. Available from: https://www.who.int/ ncds/surveillance/steps/Rwanda_2012_STEPS_Report.pdf.

17. Armitage LC, Whelan ME, Watkinson PJ, et al. Screening for hypertension using emergency department blood pressure measurements can identify patients with undiagnosed hypertension: a systematic review with meta-analysis. J Clin Hypertens. 2019; 21: 1415-25. https://doi.org/10.1111/jch.13643 PMID: 31385426

18. Fryar $\mathrm{CD}$, Ostchega $\mathrm{Y}$, Hales $\mathrm{CM}$, et al. Hypertension prevalence and control among adults: United States, 2015-2016. Hyattsville, MD: National Center for Health Statistics; 2017. Report No.:NCHS data brief, no 289 .

19. World Health Organization. Hearts: technical package for cardiovascular disease management in primary health care [Internet]. Geneva, Switzerland: World Health Organization; 2016 [Cited 2020 June 1]. Available from: https://apps.who.int/iris/bitstream/handle/10665/252661/9789241511377-eng.pdf? sequence $=1$.

20. van de Vijver S, Akinyi $\mathrm{H}$, Oti S, et al. Status report on hypertension in Africa-consultative review for the 6th ession of the African Union Conference of Ministers of Health on NCD's. Pan Afr Med J. 2013; 16: 38. https://doi.org/10.11604/pamj.2013.16.38.3100 PMID: 24570798

21. Chiang W, Jamshahi B. Asymptomatic hypertension in the ED. Am J Emerg Med. 1998; 16: 701-4. https://doi.org/10.1016/s0735-6757(98)90181-4 PMID: 9827753

22. Bukhman G, Mocumbi AO, Horton R. Reframing NCDs and injuries for the poorest billion: a Lancet Commission. The Lancet 2015; 386: 1221-2. https://doi.org/10.1016/S0140-6736(15)00278-0 PMID 26403914

23. The Lancet NCDI Poverty Commission. NCDI Poverty News \& Updates. Countries invited to establish national NCDI Poverty Commissions or to implement PEN-Plus services for severe NCDs [Internet]. The Lancet NCDI Poverty Commission; 2020 [Cited 2020 May 1]. Available from: http://www. ncdipoverty.org/blog/2020/national-commission-and-pen-plus-rfis.

24. GBD 2015 Tobacco Collaborators. Smoking prevalence and attributable disease burden in 195 countries and territories, 1990-2015: a systematic analysis from the Global Burden of Disease Study 2015 Lancet 2017; 389: 1885-906. https://doi.org/10.1016/S0140-6736(17)30819-X PMID: 28390697

25. Collins SE. Associations between socioeconomic factors and alcohol outcomes. Alcohol Res. 2016; 38 : 83-94. PMID: 27159815

26. Brody A, Rahman T, Reed B, et al. Safety and efficacy of antihypertensive prescription at emergency department discharge. Acad Emerg Med. 2015; 22: 632-5. https://doi.org/10.1111/acem.12660 PMID: 25904073

27. World Health Organization. WHO guideline on health policy and system support to optimize community health worker programmes [Internet]. Geneva, Switzerland: World Health Organization; 2018 [Cited 2020 June 1]. Available from: http://apps.who.int/iris/bitstream/handle/10665/275474/9789241550369eng.pdf?ua $=1$.

28. Ngaruiya C, Oti S, van de Vijver S, et al. Target women: Equity in access to mHealth technology in a non-communicable disease care intervention in Kenya. PLoS One 2019; 14: e0220834. https://doi.org/ 10.1371/journal.pone.0220834 PMID: 31509540

29. World Health Organization. The SAFER technical package: five areas of intervention at national and subnational levels [Internet]. Geneva, Switzerland: World Health Organization; 2019 [cited 2020 June 1]. Report No.:CC BY-NC-SA 3.0 IGO. Available from: https://apps.who.int/iris/handle/10665/330053.

30. World Health Organization. Protection from exposure to second-hand tobacco smoke: policy recommendations [Internet]. Geneva, Switzerland: World Health Organization; 2007 [Cited 2020 June 1]. Available from: https://apps.who.int/iris/handle/10665/43677. 